\title{
Chemical bond manipulation for nanostructure integration on wafer scale
}

\author{
K PRABHAKARAN* and T OGINO \\ NTT Basic Research Laboratories, 3-1 Morinosato Wakamiya, Atsugi-shi, Kanagawa-ken, 243-0198 Japan
}

\begin{abstract}
In this paper, we have briefly summarized our activity in the area of chemical bond manipulation for the integration of nanostructures on a full wafer scale. Chemical bond manipulation involves a judicious combination of surface phenomena: reactions or diffusion, and growth process such as molecular beam epitaxy (MBE). Here, we present our results on oxidation, metallization and nitridation and their role in the formation of nanostructures. We find that oxygen changes the bonding partner from $\mathrm{Ge}$ to $\mathrm{Si}$ and this phenomenon can be controlled by controlling the annealing temperature. We have employed this phenomenon for the fabrication of novel, multiperiod $\mathrm{Si}_{\mathrm{SiO}} / \mathrm{Ge}$ layered structure which exhibits interesting light emitting properties. Further, by making use of selective diffusion of cobalt atoms through Ge layers it is possible to incorporate metallic features into Ge quantum dots. Moreover, it is possible to fabricate Si nanopillars through high temperature reaction of nitric oxide. NO molecules dissociate on the surface and nitrogen atoms thus produced form nitride islands. These islands act as protective masks for the etching of Si by the oxygen atoms, through the desorption of SiO species. Occurrence of these two simultaneous processes result in the formation of nanometre-sized Si pillars capped by silicon nitride. All these results emphasize the fact that we can extend information obtained through traditional surface science experiments for the fabrication of novel structures on a full wafer scale.
\end{abstract}

Keywords. Chemical bond manipulation; nanostructure integration; wafer scale.

\section{Introduction}

Semiconductor nanostructure formation in general and that of $\mathrm{Si}$ in particular have been gaining a lot of attention by the theoreticians as well as by the experimentalists. The main thrust is to explore new avenues of application, such as in optical devices and quantum effect devices (Fisher and Chow 1993; Shen et al 1995; Ashoori 1996). Further, these can also find application as photonic crystals (Yablonovitch et al 1991), if these structures could be fabricated in a three-dimensionally periodic fashion. In the area of fabrication of nanostructured materials, control of atomic processes is the key to achieve formation of the desired species. Several researchers have reported (Avouris et al 1993) nanostructure fabrication by atom manipulation, such as using scanning tunneling microscope tip. However, this is limited to only a very small area of the wafer and moreover it involves only a single type of material. Thus, this approach is technologically not feasible. In our laboratory, we have been working on various ways to control the formation of nanostructures by suitably modifying the substrates (Ogino et al 1996). Another approach to achieve formation of nanostructures is by controlling surface phenomena, i.e. chemical reactions.

*Author for correspondence
We have termed this approach as chemical bond manipulation involving a judicious combination of surface phenomena and growth (Prabhakaran and Ogino 1997). This is schematically represented in figure 1 . The main advantage of this is the possibility to achieve formation of the desired structures on the entire surface of the wafer. The important step is to identify the reaction pathways, any selectivity with regard to surface bond formation, bond cleavage and desorption, which are usually controlled by the thermodynamics and kinetics of the system. However, the main drawback is the poor controllability of the size and distribution which to some extent can be overcome by modifying the substrate (Ogino et al 1996) structure.

\section{Experimental}

We have used a variety of $\mathrm{Si}$ and Ge wafers, differing in conductivities. These are cleaned by thermally decomposing an ultrathin ex situ prepared chemical oxide (Ishizaka and Shiraki 1986; Prabhakaran et al 1994a), inside the UHV chamber. In order to ensure good quality starting substrates, we deposited buffer layers $(\sim 100$ $200 \AA$ ) on these samples. The UPS and XPS measurements were performed using an electrostatic analyzer (VG CLAM II, equipped with three channeltrons) with $\mathrm{He} \mathrm{I}$ and $\mathrm{Al} / \mathrm{Mg} \mathrm{K} \alpha$ sources, respectively, in the normal 
emission geometry. All the spectroscopic measurements were carried out at room temperature. NO gas was leaked on to the samples from a gas bottle $(50 \mathrm{cc})$ attached to the UHV chamber through a needle valve. Ultra dry $\mathrm{O}_{2}$ (moisture content less than $100 \mathrm{ppb}$, as monitored by a hygrometer attached on line) was leaked into the chamber from a main line through a needle valve. In the case of etching with oxygen, molecular oxygen was dosed onto the nitrided surface at a temperature of $850^{\circ} \mathrm{C}$. Medium energy ion scattering (MEIS) measurements were carried out in situ to understand the atomic movements taking place during surface reactions (results not presented in this paper). Sample morphology was examined, outside the UHV chamber, using a DI AFM. In addition, we performed photoluminescence (PL), cross sectional transmission electron microscopy (XTEM), scanning electron microscope (SEM) and LEED measurements. Solid source MBE of $\mathrm{Si}$ and $\mathrm{Ge}$ was carried out in a separate chamber attached to the analysis chamber. We ensured quality of the epitaxial layers by monitoring the reflection high energy electron diffraction (RHEED). Metal deposition (cobalt) was performed from a high temperature Knudson cell, from a $\mathrm{BeO}$ crucible operated at $1200^{\circ} \mathrm{C}$. We also performed scanning Auger microscopic measurements (spatial resolution of the order of $\sim 100 \mathrm{~nm}$ ) to determine the atomic concentrations.

\section{Results and discussions}

\subsection{Fabrication of novel nanometre sized multilayer} structures through a combination of surface reaction and growth

There have been several attempts to improve the performance of Si-based devices, such as hetero-junction bipolar transistors and modulation-doped field effect transistors, by using SiGe alloys (People 1986; Iyer et al

Atom Manipulation:

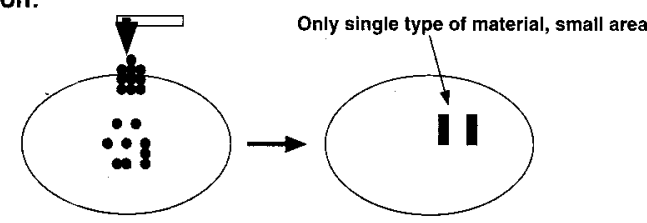

Chemical Bond Manipulation :

A Judlcious Combination of MEE and Surface Phenomenon

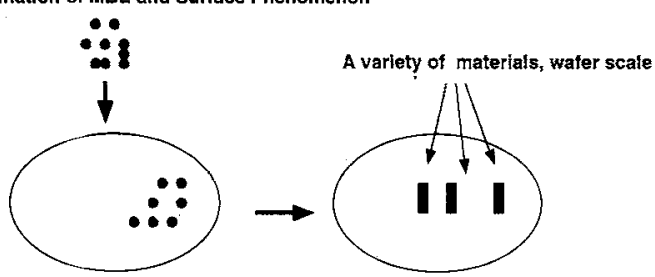

Figure 1. Schematic description of the chemical bond manipulation and comparison with atom manipulation with STM tip.
1989; Park et al 1989). Since one of the most advantageous features in Si devices is the availability of good quality oxide, prepared by thermal oxidation and its interface with substrate, control of oxidation process is a key technology for future SiGe-based devices. It has been reported that during $\mathrm{SiGe}$ alloy oxidation, a $\mathrm{SiO}_{2}$ layer is preferentially formed and $\mathrm{Ge}$ is rejected into the $\mathrm{SiO}_{2} / \mathrm{SiGe}$ interface. However, most of these studies have been carried out in furnaces and on thick layers of SiGe alloys (Fathy et al 1987; Patton et al 1988; LeGoues et al 1989; Nayak et al 1990; Liou et al 1991). In order to control the interface characteristics we believe that in situ studies are essential to understand the mechanism of the initial oxidation process. Sometime ago we reported our (Prabhakaran et al 1993, 1994b) study on the early stages of oxidation of a thin layer of $\mathrm{Ge}$ grown on $\mathrm{Si}(001)$ surface. We found that oxygen changes its bonding partner from $\mathrm{Ge}$ to $\mathrm{Si}$ on increasing the temperature. Further, surface sensitive experiments using synchrotron radiation revealed that such a reaction takes place in two different steps. The first step involves the breakage of $\mathrm{Ge}-\mathrm{O}$ bonds and formation of $\mathrm{Si}-\mathrm{O}$ bonds. This process continues until the germanium oxide is completely reduced to elemental Ge. The second step involves rearrangement of oxygen atoms around $\mathrm{Si}$ to form the stable stoichiometric $\mathrm{SiO}_{2}$. Figures $2 \mathrm{a}$ and $\mathrm{b}$ describe these results. The attenuation of the oxide signal in $\mathrm{Ge} 3 d$ and its concomitant increase in $\mathrm{Si} 2 p$ unequivocally establishes the occurrence of the bonding partner change reaction. The principle of manipulation of surface chemical bonds in this case can be explained on the basis of the large differences in the heats of formation (CRC Handbook of Chemistry and Physics 1989) of $\mathrm{Ge}$ oxides (for $\mathrm{GeO}$ and $\mathrm{GeO}_{2}, \Delta \mathrm{H}_{\mathrm{f}}=-62$ and $-131 \mathrm{kcal} / \mathrm{mol}$, respectively) compared to that of Si oxide $\left(\Delta \mathrm{H}_{\mathrm{f}}=-217 \mathrm{kcal} / \mathrm{mol}\right)$. The same factor is reflected in the strength of the chemical bonds ( $\mathrm{Ge}-\mathrm{O}$ and $\mathrm{Si}-\mathrm{O}$, 151 and $191 \mathrm{kcal} / \mathrm{mol}$, respectively).

The above-mentioned experiments were performed on $\mathrm{Si}(001)$ wafers and the occurrence of bonding partner change reaction resulted in considerable surface roughening as seen by the formation of nanometre sized voids. Recently, we carried out similar experiments on $\mathrm{Si}(111)$ surface, which has lower surface energies (Prabhakaran et al 1998). On a clean $\mathrm{Si}(111)$ sample, 4 monolayers $(\sim 6.5 \AA)$ of $\mathrm{Ge}$ were deposited at room temperature (RT). The sample was oxidized by exposing to flowing oxygen, outside the ultrahigh vacuum (UHV) chamber, for one min. This process resulted in the formation of nearly two monolayers of $\mathrm{Ge}$ oxide. The sample was re-inserted into the UHV chamber immediately and characterized using photoemission measurements. Si (3 nm) was deposited onto this sample at RT. This procedure (Ge deposition, oxidation and $\mathrm{Si}$ deposition) was repeated seven times (in the case of the sample 
discussed in this paper, hereafter called as "7-period sample'), each time characterizing the surface species. Finally, the sample was capped with $\mathrm{Ge}(6.5 \AA)$ and $\mathrm{Si}$ $(5 \mathrm{~nm})$. The final structure of the sample consisted of

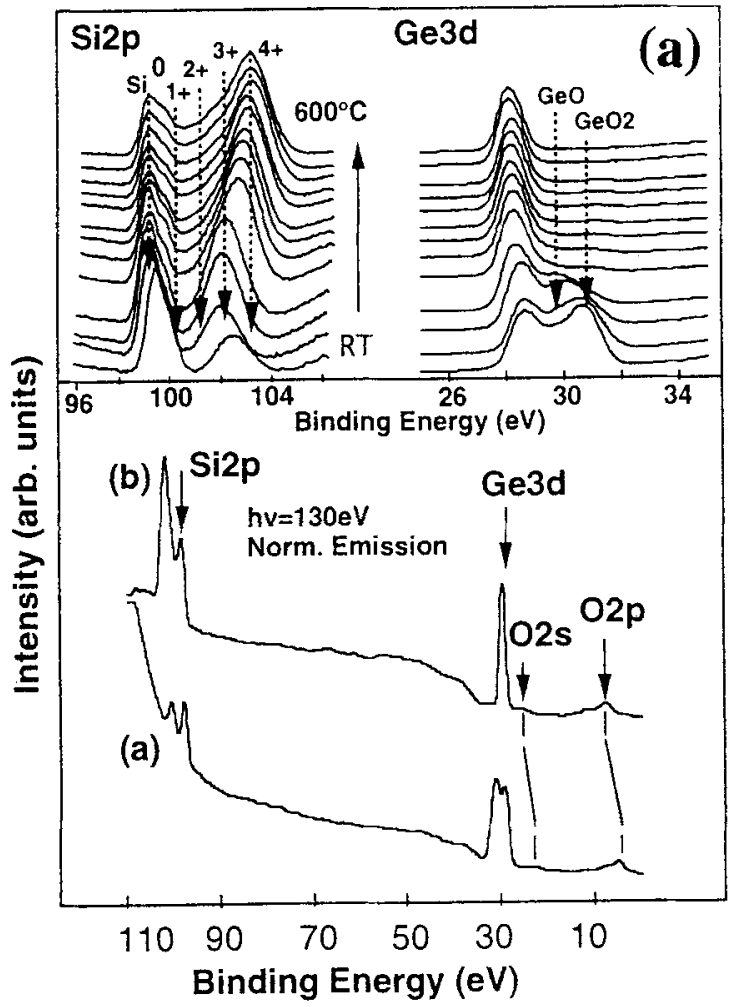

(b)

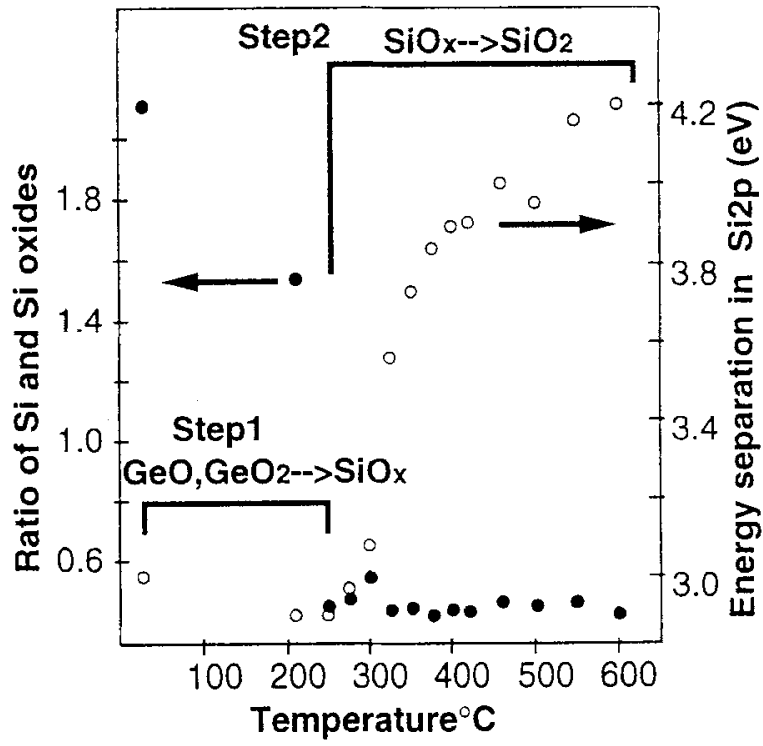

Figure 2. (a) SRPES wide scan of (a) air oxidized $5 \AA \mathrm{Ge}$ deposited on $\mathrm{Si}(100)$ and (b) annealed to $600^{\circ} \mathrm{C}$. Inset shows the $\mathrm{Si} 2 p$ regions recorded as a function of temperature. (b) Variation of the ratio of elemental Si $2 p$ to that of the oxides. Right axis shows the energy separation between the peak positions due to elemental $\mathrm{Si}$ and that due to the combined oxides in Si $2 p$ spectra. The plot shows two distinct steps in the bonding partner change reaction. ultrathin silicon oxide layers sandwiched between ultrathin $\mathrm{Si}$ and Ge layers as described later on.

Figure 3 a shows the XPS in the Ge $2 p$ region after oxidizing the Ge covered Si(111) surface (spectrum (a)), and after depositing Si onto the Ge oxide covered surface (spectrum (b)). Spectrum (a) shows that oxidation results in the formation of a mixture of $\mathrm{Ge}$ oxides. On depositing $\mathrm{Si}$ onto this surface, $\mathrm{Ge}-\mathrm{O}$ bonds are broken and $\mathrm{Si}-\mathrm{O}$ bonds are formed and therefore the signal due to the oxides in $\mathrm{Ge} 2 p$ spectrum is attenuated and returns to that of the sample prior to oxidation, implying complete
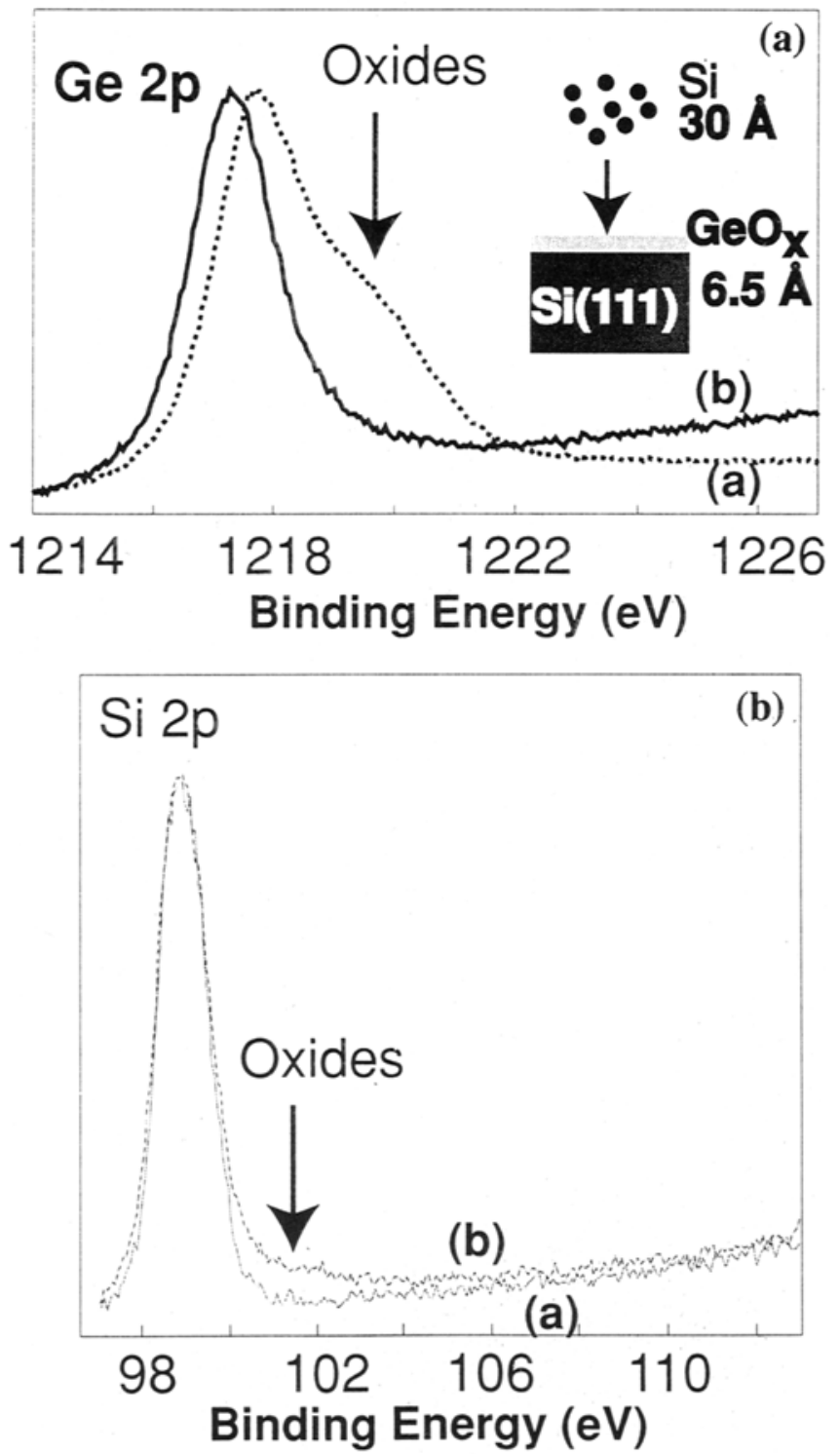

Figure 3. XPS in the Ge $2 p$ region from a thin (4ML) Ge layer deposited on $\mathrm{Si}(111)$ and oxidized (spectrum (a)). Spectrum (b) is obtained after depositing $20 \AA \mathrm{Si}$ onto this sample at RT, indicating the disappearance of germanium oxide peaks. The height has been normalized to make the comparison clearer. Inset shows the corresponding $\mathrm{Si} 2 p$ spectra. Signal due to silicon oxide formation on depositing $\mathrm{Si}$ onto the germanium oxide covered surface is indicated. 
reduction (see spectrum (b)). Corresponding Si $2 p$ spectra are plotted in figure $3 \mathrm{~b}$ and show signal due to silicon oxides after the deposition of $\mathrm{Si}$ onto the $\mathrm{Ge}$ oxide covered $\mathrm{Si}(111)$ surface. The signal due to the oxides in $\mathrm{Si} 2 p$ is weak compared to the $\mathrm{Ge}$ oxides because the main peak is dominated by bulk Si. Spectral changes in $\mathrm{O} 1 s$ as well as UPS corroborate such chemical bond manipulation reaction.

Figure 4 is the XTEM image from the 7-period sample, indicating the formation of continuous layers with an interface roughness of the order of atomic dimensions. Figure 4 also shows the Auger depth profiling data. The sharp dips observed in the Si LVV signal due to the depletion of elemental $\mathrm{Si}$ and the corresponding peaks in O KLL signal, show the formation of silicon oxide layers with abrupt interfaces. Figure 5 shows the morphology of the clean substrate and that of the 7-period sample, as observed by AFM, (a) and (b), respectively. It is interesting to note that despite the overlayer growth (total thickness, $\sim 30 \mathrm{~nm}$ ) and chemical bond manipulation in each period, the original wafer morphology is preserved. This is because the chemical bond manipulation is achieved at RT where the atom diffusion rates responsible for interface roughening are considerably reduced (Grossman and Schubert et al 1993). This helps to maintain a smooth interface, reduce the strain in each layer and thereby maintain surface planarity. This is a unique aspect of the chemical bond manipulation and permits the formation of the new structure on a full wafer scale.

Figure 6 shows the PL spectrum from this sample, measured using $325 \mathrm{~nm}$ excitation light from a $\mathrm{He}-\mathrm{Cd}$ laser. Blue-green luminescence is observed (visible to the naked eye) at RT with a spectral peak at $\sim 26 \mathrm{eV}$ (full width at half maximum of the order of $1 \mathrm{eV}$ ). We recorded the PL intensity as a function of temperature and found that the intensity decreases when the sample is brought to room temperature from $13 \mathrm{~K}$. Additionally, we fabricated samples with different periods (4 and 2) and found that the PL intensity from those samples was significantly low compared to the 7-period sample. We fabricated samples with varying thicknesses of $\mathrm{Si}$ and $\mathrm{Ge}$ in a similar way and performed PL measurements, in order to examine the effect of quantum confinement on the blue-green emission. We did not observe any shift in the PL peak energy and therefore, rule out the quantum confinement effect as a possible explanation for the light emission. Other possibilities for the emission are defects created at the interface or $\mathrm{SiO}_{2}$ layer (Skuja 1994). Further, we extended this work to fabricate ultrathin $\mathrm{SiO}_{2}$ layer buried between $\mathrm{Si}$ and Ge layers by oxygen implantation into $\mathrm{Si} / \mathrm{Ge}$ multilayer structures (Ogino et al 1997).

\subsection{Fabrication of $\mathrm{Ge} / \mathrm{CoSi} / \mathrm{Si}$ nanodiscs and incorporation of metallic feature into Ge quantum dot network}

Since it is important to have metallic features on semiconductors for contacts and interconnection in devices, we investigated the behaviour of cobalt atoms on $\mathrm{Si}$ and $\mathrm{Ge}$ wafers. In the literature, there is a vast amount of work reported in the area of $\mathrm{Si}$ /metal systems (von Kanel 1992). However, interaction of metals with systems involving Ge are not many (Ashburn et al 1992) and deserves a thorough investigation. From our experiments we find a distinct selectivity with regard to the onset temperature of inward diffusion as shown in figure 7 . Co atoms begin to diffuse into bulk $\mathrm{Ge}$ at lower temperatures than that in Si. The explanation of this difference in behaviour is mainly attributed to the equilibrium defect concentration and the mechanism of diffusion. By employing this phenomenon we fabricated epitaxial cobalt silicide at the interface between a thin layer of $\mathrm{Ge}$ deposited on $\mathrm{Si}$. 2ML of Co is deposited on a
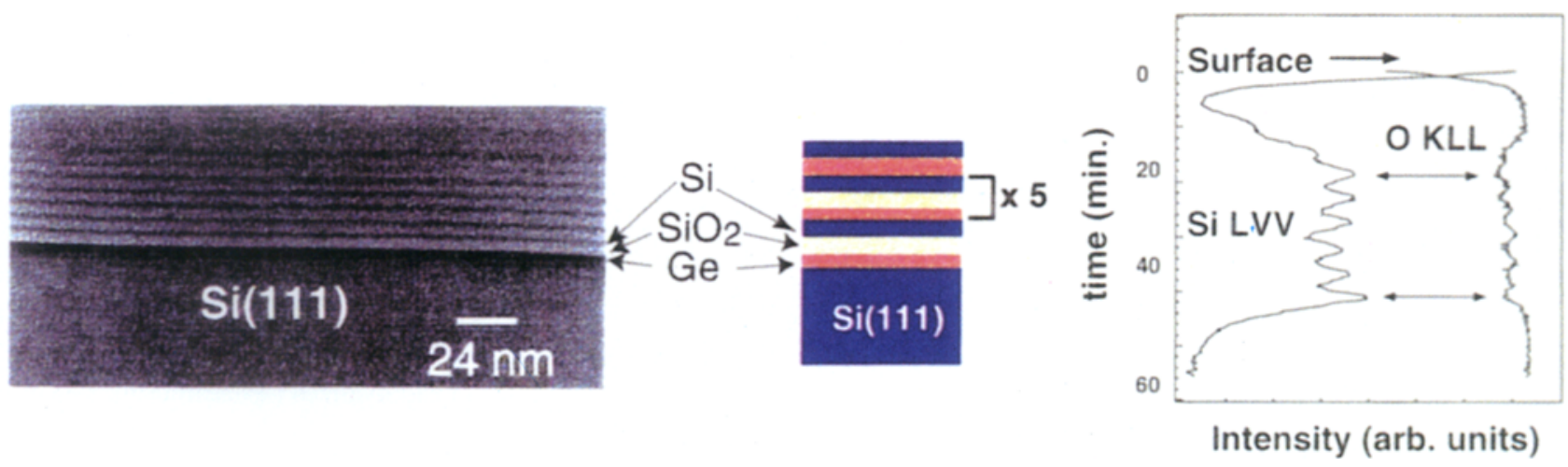

Figure 4. XTEM image from the 7-period sample showing the formation of continuous layers and sharp interfaces. A schematic description of the sample is also given. Auger depth profile data from the 7-period sample is also shown. The sharp dips in the Si LVV signal (due to depletion of elemental Si) and the corresponding peaks in O KLL signal (as indicated by the arrows), confirm the formation of silicon oxide layers, as a result of the chemical bond manipulation. 

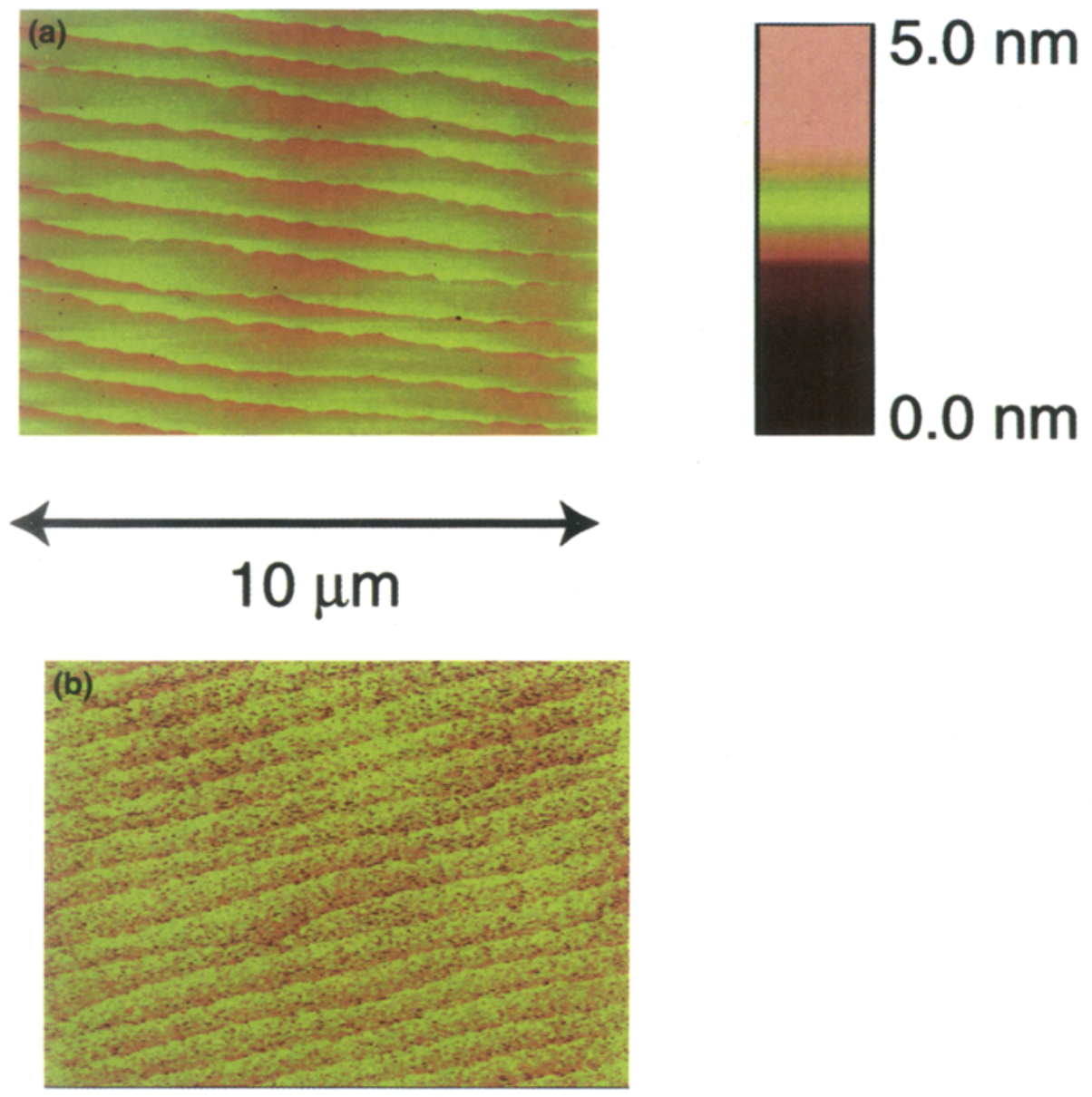

Figure 5. Surface morphology (AFM) of (a) clean $\mathrm{Si}(111)$ substrate, and (b) 7-period sample. The original surface morphology is preserved, as indicated by the clear observation of surface steps.

$\mathrm{Si}(111)$ surface, which is covered with $\sim 4 \mathrm{ML}$ of $\mathrm{Ge}$, both at room temperature. On annealing the sample at $460^{\circ} \mathrm{C}$, Co diffuses through the Ge layer and reacts with the $\mathrm{Si}$ atoms underneath. This results in the formation of a buried $\mathrm{CoSi}_{2}$ layer. XTEM pictures as well as the blocking dip pattern in MEIS suggest that the $\mathrm{CoSi}_{2}$ formed at the interface between $\mathrm{Si}$ and $\mathrm{Ge}$, is epitaxial, twinned at the interface (Prabhakaran et al 1996, 1997a). There is significant atomic movement associated with the diffusion and reaction resulting in the formation of nanometre-sized discs. Figure 8 shows the XTEM showing the lattice image as well as the nanodiscs formed in this reaction. Co layer undergoes agglomeration and form $\mathrm{Ge} / \mathrm{CoSi}_{2} / \mathrm{Si}$ dot structures and $\mathrm{Ge} / \mathrm{Si}$ terraces. A mixture of $1 \times 1$ and $5 \times 5$ patterns originating respectively from $\mathrm{Ge} / \mathrm{CoSi}_{2} / \mathrm{Si}$ and $\mathrm{Ge} / \mathrm{Si}$ regions are observed in RHEED and LEED. These results suggest a new way of fabrication of buried epitaxial metallic layers and semiconductor/ metal nanostructures.

It is well known that growth of $\mathrm{Ge}$ on $\mathrm{Si}$ follows the Stranski-Krastanow mode in which about 4 monolayers of $\mathrm{Ge}$ form a wetting layer, and if deposited in excess they form islands. In our group, we have developed a technique by which we can control the spatial distribution of these Ge quantum dots and it is possible to have an ordered arrangement (Hibino and Ogino 1995). By utilizing the selective diffusion of $\mathrm{Co}$ atoms through these dots, we can incorporate metallic features into these quantum dots. Figure 9a shows the SEM picture of such a Ge quantum dot network. Figure $9 \mathrm{~b}$ shows the variation of atomic concentration of $\mathrm{Ge}$ and $\mathrm{Co}$ on the wetting layer as well as on the dots monitored using SAM as a function of $\mathrm{Ar}$ ion sputtering. As can be seen, the Co and $\mathrm{Ge}$ signals from the wetting layer decrease and finally disappear as the sputtering proceeds. The $\mathrm{Ge}$ signal from the top of the quantum dot also decreases because of the removal of the top few layers. However, the Co signal shows only a marginal decrease and maintains a constant value. These results in combination with XPS, UPS and XTEM results clearly indicate that the dots contain metal atoms in the form of silicide. Photoemission as well as XTEM support this view. It is to be remembered that traditional methods such as ion implantation for metallization, fail in the nanoregime 
as the beam damage itself is of the order of several hundred nanometres. The process described here is schematically represented in figure 10 .

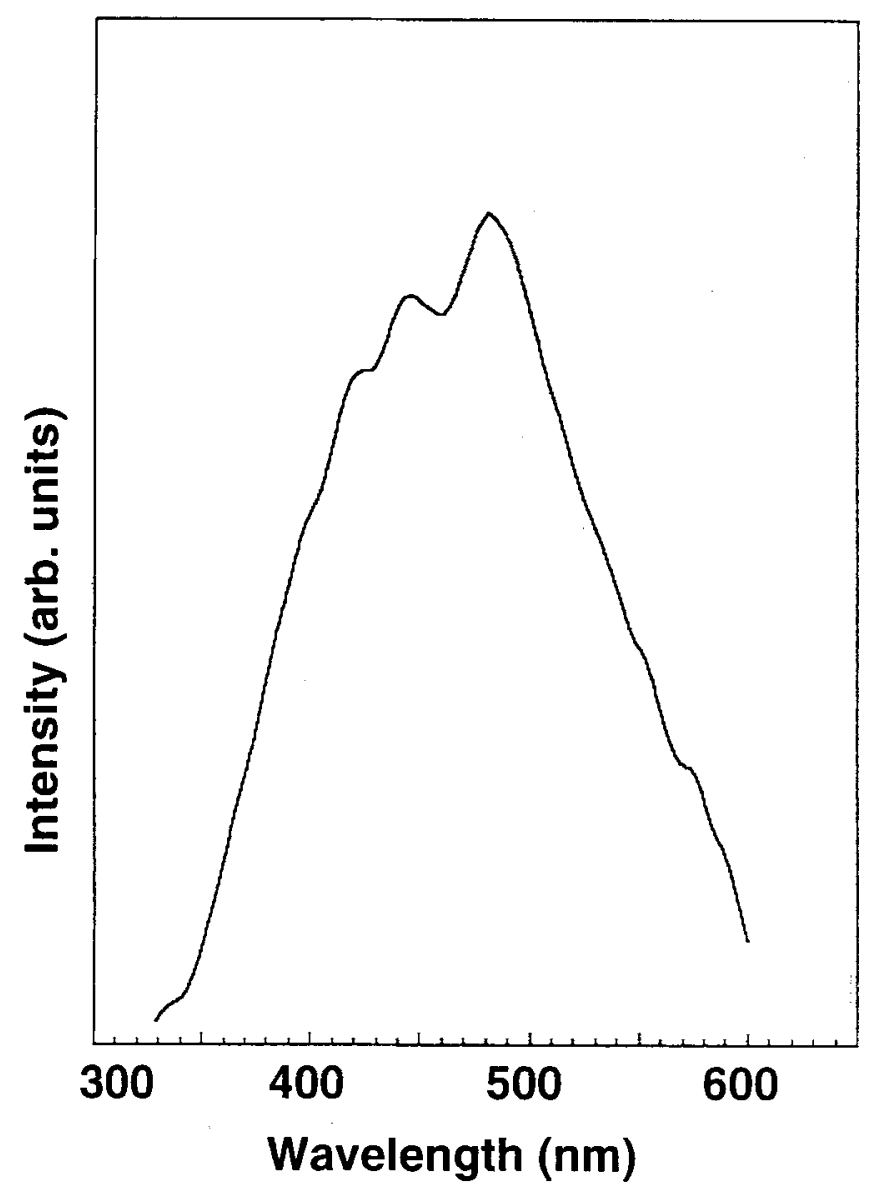

Figure 6. RT PL spectrum from the 7-period sample excited by $\mathrm{He}-\mathrm{Cd}$ laser $(325 \mathrm{~nm}, \sim 2 \mathrm{~mW})$

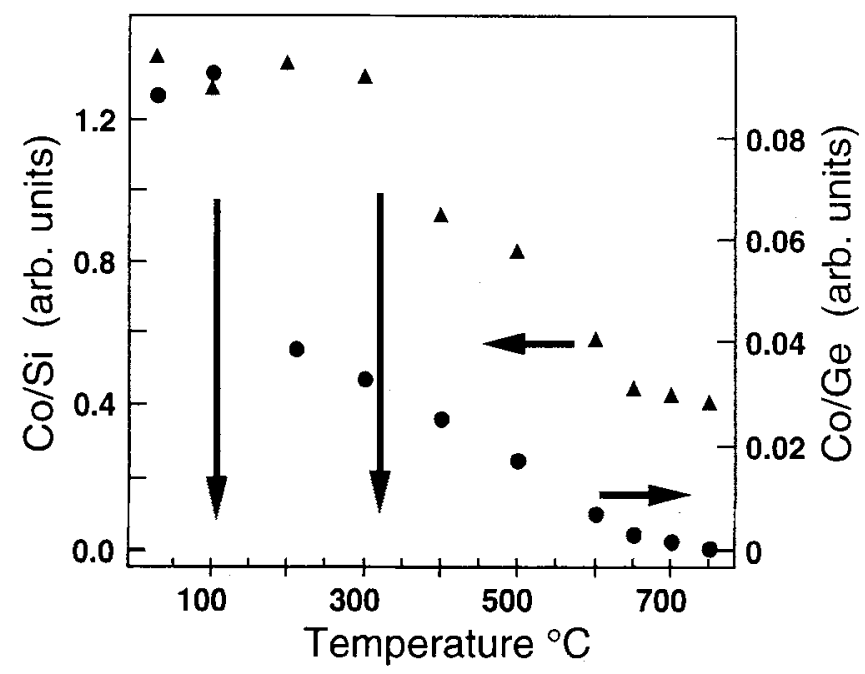

Figure 7. Temperature dependence of $\mathrm{Co}$ concentration, as obtained from the XPS core level spectra, for 1.2 ML of Co deposited at room temperature on $\mathrm{Si}(100)$ and $\mathrm{Ge}(100)$. The plot shows a distinct selectivity with regard to the onset temperature of inward diffusion.

\subsection{Fabrication of Si nanopillars through high} temperature reaction of nitric oxide with $S i$ wafer

Several researchers have reported oxynitridation of $\mathrm{Si}$ surfaces using NO gas (Hegde et al 1995; Lu et al 1996; Kamath et al 1997). We have employed this reaction in a novel way to fabricate $\mathrm{Si}$ nanostructures. Here, we describe the fabrication of Si nanopillars through the high temperature reaction of NO with $\mathrm{Si}$ surfaces. Figure 11 gives a schematic representation of the principle behind the process of Si nanopillar formation. When NO molecule approaches the surface, it dissociates and nitrogen atoms stick to the surface forming nitride islands. Since the substrate temperature is high $\left(\sim 850900^{\circ} \mathrm{C}\right)$, oxygen atoms etch off the surface $\mathrm{Si}$ atoms through the desorption of $\mathrm{SiO}$ species and nitride islands act as protective masks for this etching process. Silicon nitride is stable on the surface upto relatively higher temperatures $\left(\sim 1200^{\circ} \mathrm{C}\right)$. It is to be noted that both the island formation and the etching processes take place simultaneously. Continuation of both the processes by long exposure to $\mathrm{NO}$ gas, result in the formation of nanometre-sized Si pillars capped by nitride islands. The advantage with

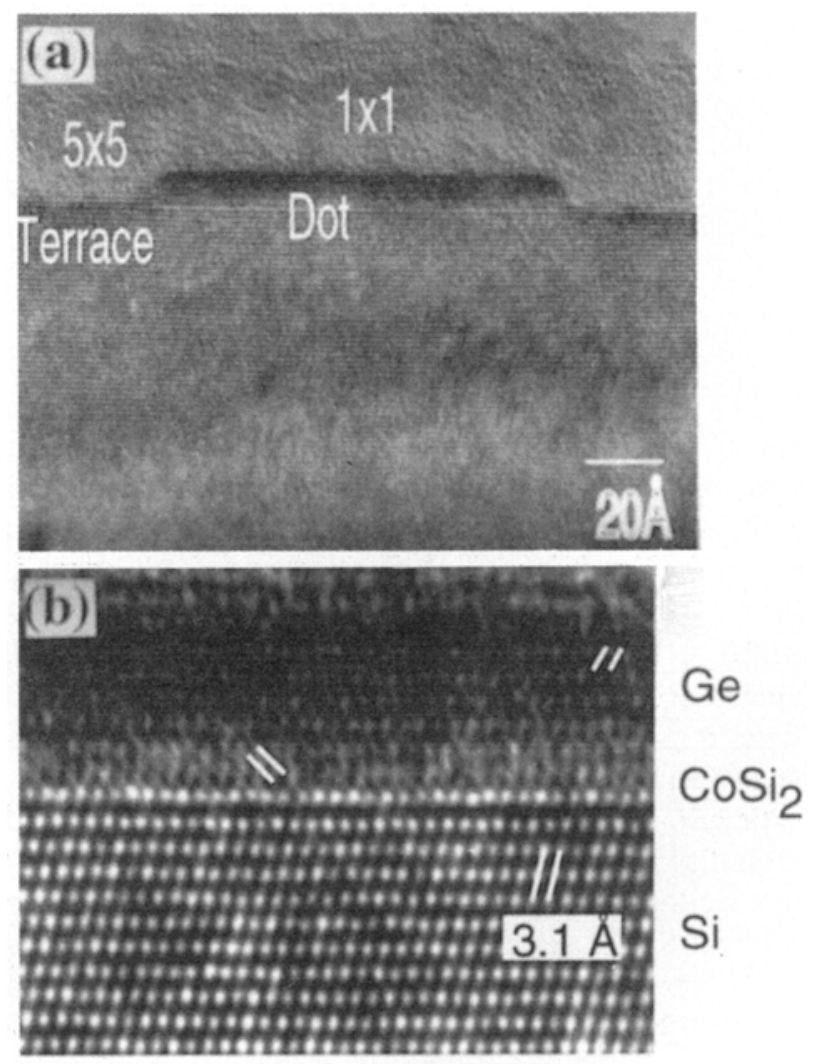

Figure 8. Cross sectional TEM of the $\operatorname{Co}(1 \cdot 2 \mathrm{ML}) / \mathrm{Ge}(4 \mathrm{ML}) /$ $\mathrm{Si}(111)$ after annealing at $460^{\circ} \mathrm{C}$, showing the formation of flat terraces and nanodisc/dot structures. The bottom figure is the expansion of the disc structure showing the lattice images and indicate the epitaxial cobalt silicide layer formation. 
this single step process is that the nitride capping also grows thicker and therefore its protective action is significantly enhanced and even becomes resistant to chemical etching performed outside the UHV. The region between the pillars seem to be preferentially etched by the oxygen rather than get nitridized during the later stages of the reaction. This selectivity may be linked to the thermodynamics and kinetics of the two competitive processes. Figure 11 also shows the AFM in the initial stages of the etching mode reaction on $\mathrm{Si}(111)$. Corroded step edges can be observed where the step edges are irregular (due to partial etching) and this phenomenon

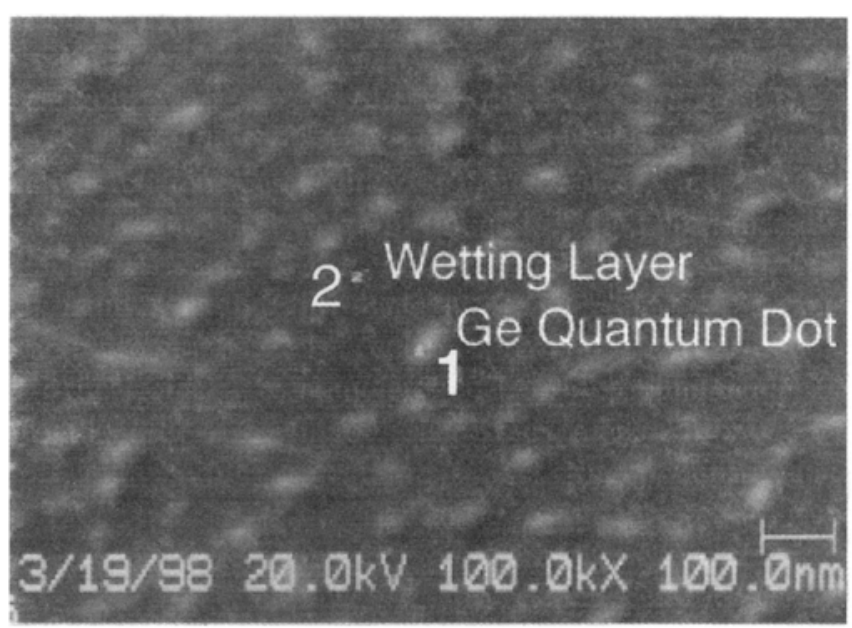

$\mathrm{Co} / \mathrm{Ge}(\mathrm{QD}) / \mathrm{Si}(111)$

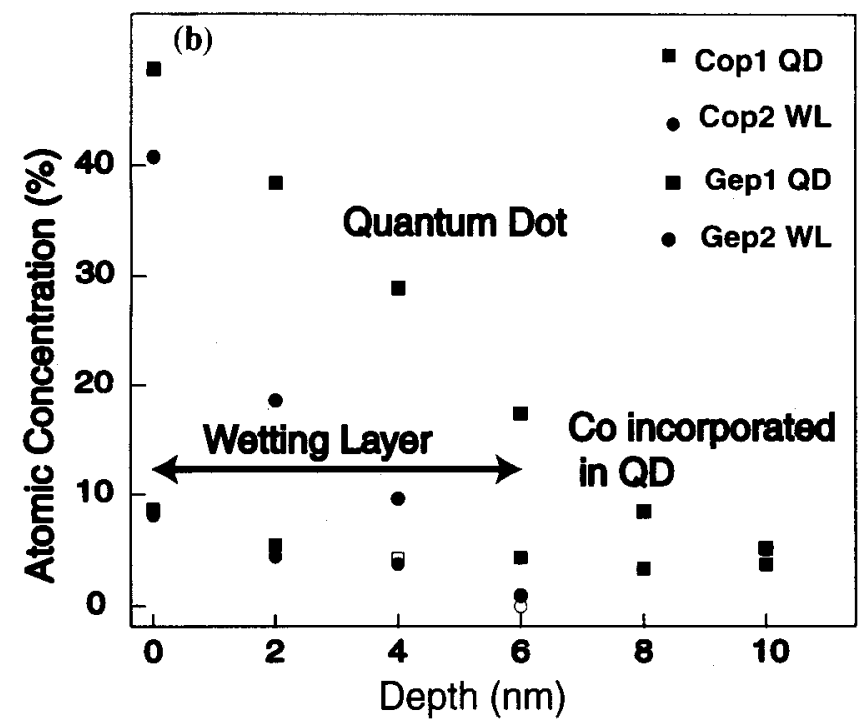

Figure 9. (a) SEM picture of Ge quantum dot network showing the dots and wetting layer and (b) Auger signals were recorded selectively from these areas and plotted in the figure below. The plot shows that $\mathrm{Co}$ atoms can be incorporated into the Ge dots through selective diffusion. seems to be similar to the step flow growth occurring in the case of homoepitaxy of $\mathrm{Si}$. This means that atom attachment/detachment process takes place selectively at step edges. The AFM image clearly indicates that the etching process starts at the step edges and is sporadically pinned by the formation of nitride islands at step edges.

AES, UPS, XPS and LEED measurements were performed to ascertain the nature of the surface species. The clean surfaces are characterized by the presence of sharp emission from the dangling bond states of the atoms in the termination layer. After the reaction surface states continue to be observed with diminished intensity, and clear peaks $(\sim 6.4 \mathrm{eV}$ due to $\mathrm{N} 2 p$-derived orbitals) due to surface nitride species are also observed. This clearly indicates the formation of two different domains consisting of bare $\mathrm{Si}$ area and the Si nanopillars capped by the nitride species. In the AES, strong signals due to surface nitride species are observed at kinetic energies $84 \mathrm{eV}$ and $387 \mathrm{eV}$. Corresponding XPS spectra in the Si $2 p$ region shows a clear signal chemically shifted by $\sim 2.8 \mathrm{eV}$ typical of the formation of $\mathrm{Si}_{3} \mathrm{~N}_{4}$ type of nitride and the $\mathrm{N} 1 s$ is observed at $398 \mathrm{eV}$ (Tabe and Yamamoto 1996). One important difference between (111) and (100) surfaces is the nature of the $\mathrm{N} 2 p$-derived peak in the He I UPS. The peak is rather narrow in the case of (111) surface and this, we believe, is due to the better ordering in the nitride layer. For the same reason, the signal due to nitride in the Si $2 p$ spectrum is broad in the case of (100) compared to that in (111). The LEED observation also substantiates this view. In the case of (111) surface, during the early stages of the reaction with NO, the $7 \times 7$ pattern becomes diffuse. However, as nitrogen coverage increases ( $1 \mathrm{ML})$ a mixture of $7 \times 7$ and a quadruplet structure emerges. There is some evidence for an $8 \times 8$ pattern also at this stage. At higher coverages (2-3 ML), we observe sharp pattern consisting of $1 \times 1$ and quadruplet structures. In the case of (100) surface, at higher nitrogen coverages, a diffuse $1 \times 1$ pattern is observed.

Figure 12 shows the typical AFM image after the high temperature reaction with $\mathrm{NO}$ in the case of (111) and (100) surfaces. The images clearly show high density pillar formation in both the cases. The nature of the pillars and their interaction between one another appears to be quite sensitive to the surface temperature. For example, images (b) and (c) in figure 13 are recorded from different portion of the same sample, with the difference that the attained temperature was slightly higher $\left(-25^{\circ} \mathrm{C}\right)$ for the portion imaged in (c) compared to that in image (b). As seen in the AFM images, the pillars seem to undergo coalescence giving rise to a kind of nanometer-sized $\mathrm{Si}$ walls. We are performing other experiments to understand this phenomenon.

Figure 13 depicts that the height of these pillars can be increased by performing further etching of Si sepa- 


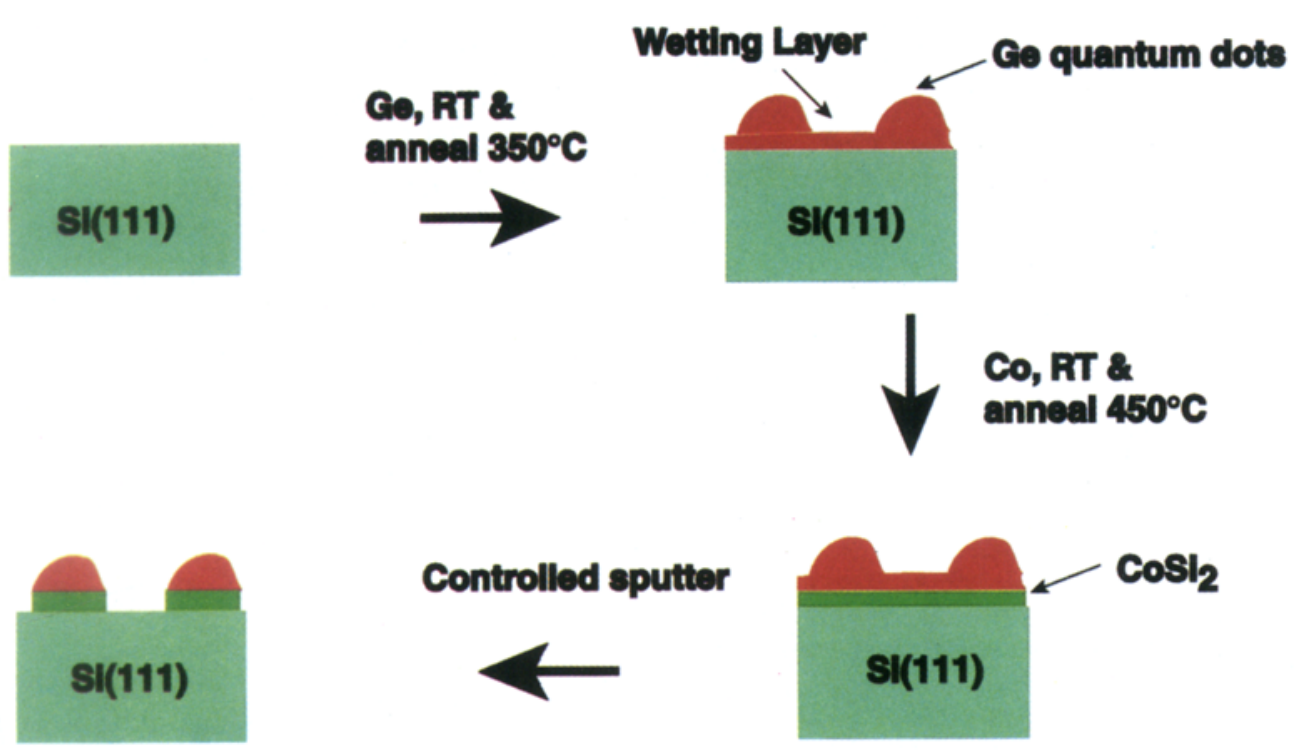

Figure 10. Schematic description of the process to incorporate metal into Ge quantum dots.
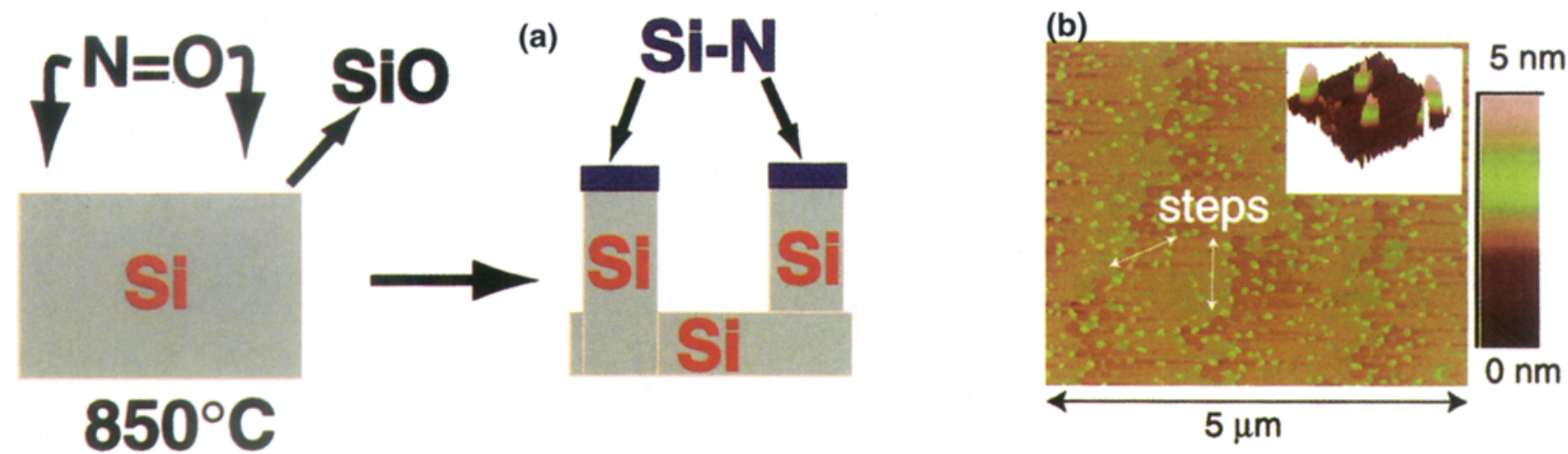

Figure 11. (a) Schematic of the high temperature reaction of NO with Si surface, which results in the formation of Si nanopillars capped by nitride islands and (b) is an AFM image showing the morphology in the early stage of the reaction (10 L NO at $850^{\circ} \mathrm{C}$ ). Surface steps become irregular in the etching process.
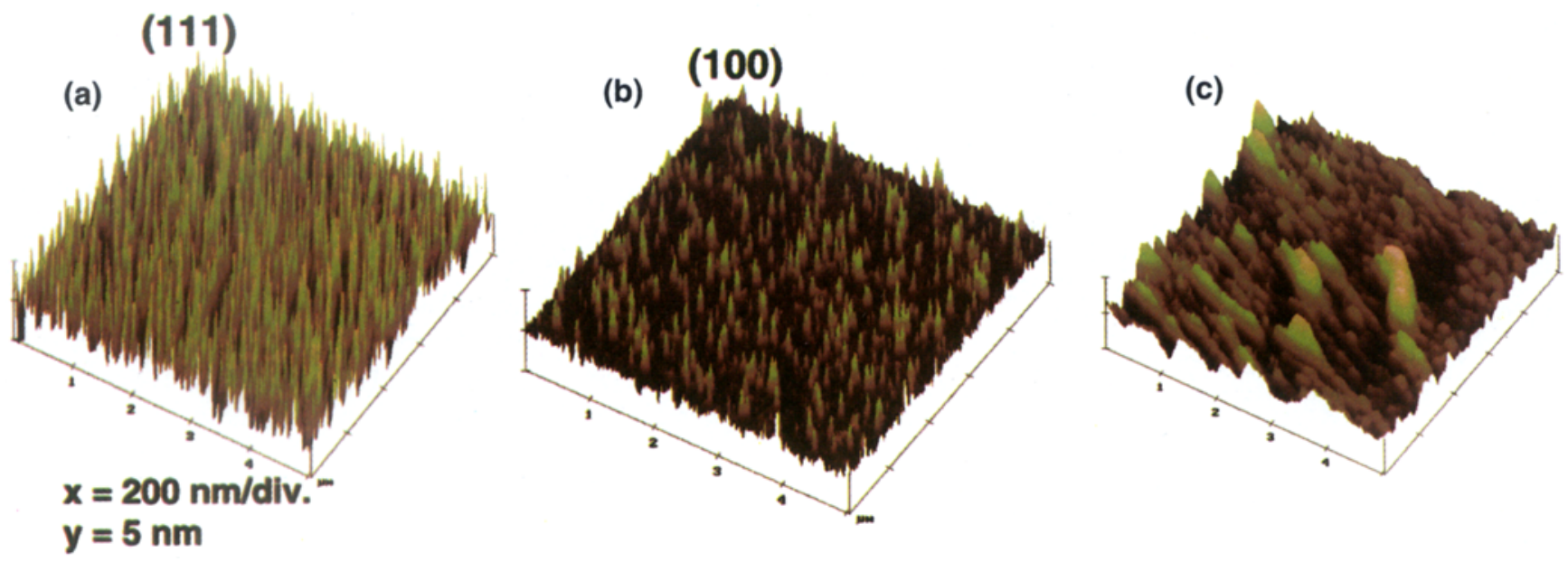

Figure 12. AFM image after dosing (a) the Si(111), (b). The $\mathrm{Si}(100)$ surfaces with NO $\left(1 \mathrm{~h}\right.$ at $1 \times 10^{-8}$ torr) at a substrate temperature of $850^{\circ} \mathrm{C}$. (c) is recorded from a different portion of the sample, same as in $\mathbf{b}$, where, the temperature is slightly higher $\left(\sim 25^{\circ} \mathrm{C}\right)$. 

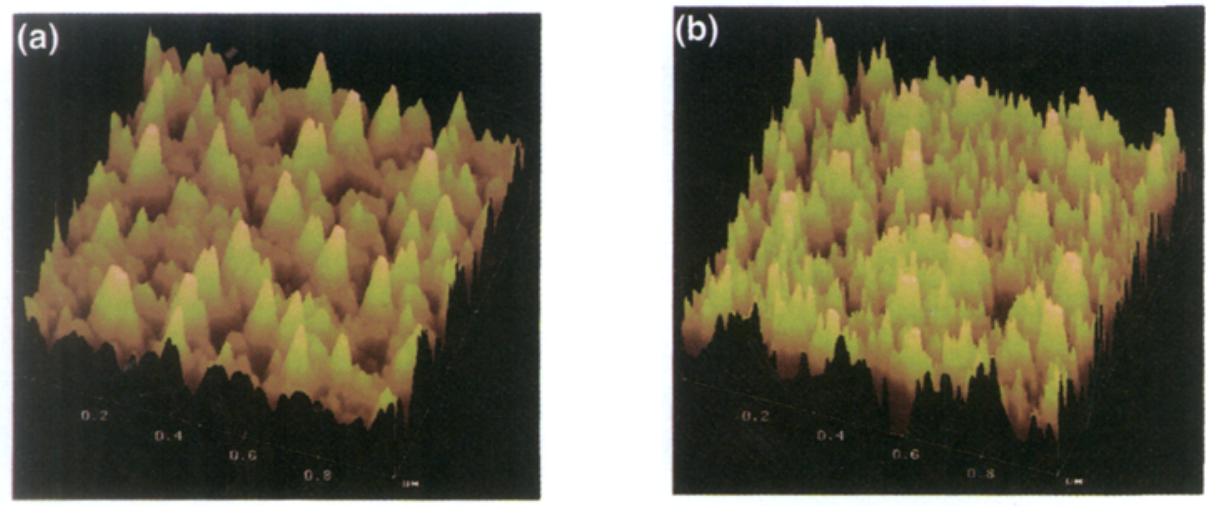

$$
X=200 \mathrm{~nm} / \text { div.; } y=\mathbf{5 m m}
$$
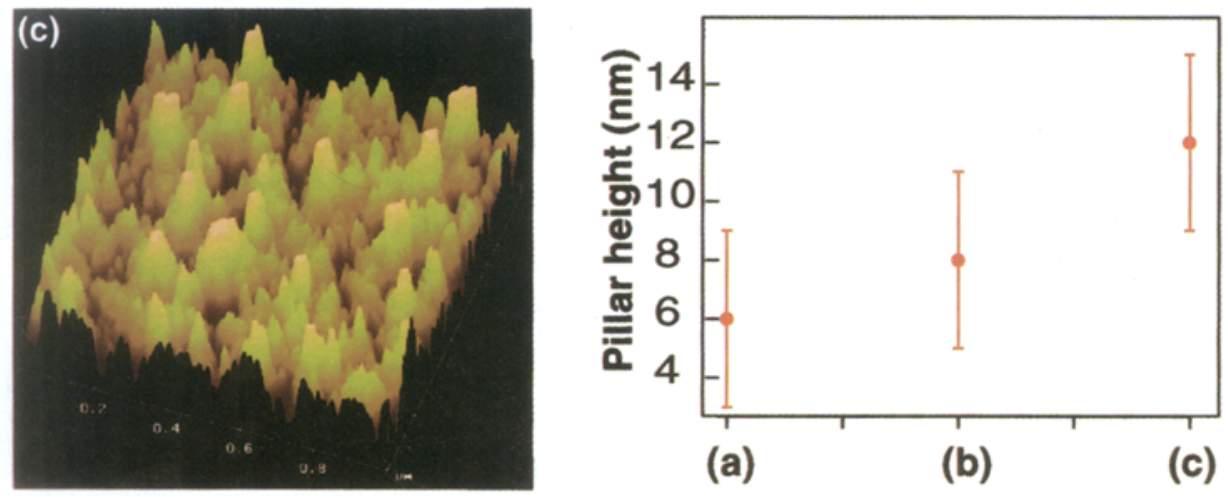

Figure 13. Experimental demonstration of the fact that the pillar height can be increased through in situ and $e x$ situ etching processes. (a) image is from a $\mathrm{Si}(100)$ exposed to $\mathrm{NO}$ at $850^{\circ} \mathrm{C}\left(1 \times 10^{-8}\right.$ torr for $\left.1 \mathrm{~h}\right)$ and subsequently etched with $\mathrm{O}_{2}$ at $850^{\circ} \mathrm{C}\left(1 \times 10^{-8}\right.$ torr for $1 \mathrm{~h}$ ). The comparison of UPS shown as an inset to figure $2 \mathrm{~b}$ correspond to this stage; (b) the same sample was taken out of the UHV chamber and etched with very dilute $(\sim 1-2 \%)$ HF for $25 \mathrm{~s}$; (c) after 3 cycles of oxidation in very dilute $(\sim 5 \%)$ $\mathrm{HNO}_{3}$ and $\mathrm{HF}$ etch; and (d) is a plot of the typical pillar height after treatments $\mathbf{a}, \mathbf{b}$ and $\mathbf{c}$.

rately. For example, the pillars formed by reaction of $\mathrm{NO}$ at $850^{\circ} \mathrm{C}$ was subjected to an etching reaction with oxygen $\left(1 \times 10^{-8}\right.$ torr for $\left.1 \mathrm{~h}\right)$ at $\sim 850^{\circ} \mathrm{C}$. After this process, the average pillar height increased by $\sim 1 \mathrm{~nm}$. It is worth noticing that the nature of the nitride species and its amount is unaffected by this etching process, as the $\mathrm{N} 1 s$ as well as $\mathrm{Si} 2 p$ signals recorded before and after the etching process were identical. Therefore, the nitride islands formed through the dissociative adsorption of NO are very stable and function effectively as a protective capping layer. Figure $13 \mathrm{~b}$ is the image of the same sample (same as (a)), which was etched ex situ in very dilute $\mathrm{HF}$. The silicon oxide formed due to the exposure to air is expected to be removed in this process. The average pillar height increased by around 1-2 nm due to the removal of $\mathrm{Si}$ from the region between the pillars. Image (c) is the same after sample was subjected to three cycles of oxidation and etching in very dilute
$\mathrm{HNO}_{3}$ and $\mathrm{HF}$, respectively. The average pillar height increased quite significantly by $3-4 \mathrm{~nm}$ after this process. Moreover, the small pillars are etched off completely, indicating that size selection is a possibility.

\section{Conclusions}

We have demonstrated chemical bond manipulation, which is a combination of surface phenomenon and growth, to fabricate novel nanostructures on a full wafer scale emphasizing thereby the role of traditional surface science in nanometre science and technology. For example, by making use of a bonding partner change reaction of oxygen, occurring on the surface, we could fabricate nanometre-sized multiperiod layered structure consisting of $\mathrm{Si} / \mathrm{SiO}_{2} / \mathrm{Ge}$ on a $\mathrm{Si}(111)$ substrate. We have also shown that it is possible to incorporate metallic features into $\mathrm{Ge}$ quantum dot network through selective 
diffusion. The main challenge in this approach is to control the size and spatial distribution of these nanostructures. We are currently working on $\mathrm{Si}$ wafers with various patterns on it to examine the reactivity difference between different regions.

\section{Acknowledgements}

It gives us great pleasure to acknowledge our colleagues Drs K Sumitomo, H Hibino, Y Kobayashi, T Fukuda, $\mathrm{H}$ Homi, T Matsumoto and Y Masumoto for their help, support and encouragement throughout the course of this work.

\section{References}

Ashburn S P, Ozturk M C, Wortman J J, Harris G, Honeycutt $\mathrm{J}$ and Maher D M 1992 J. Electronic Mater. 2181

Ashoori R C 1996 Nature 379413

Avouris Ph, Lyo I-W, Hasegawa Y 1993 J. Vac. Sci. Technol. A11 1725

CRC Hand Book of Chemistry and Physics 1989 (Boca Raton, FL: CRC Press) 1989 p. F-177

Fathy D, Holland O W and White C W 1987 Appl. Phys. Lett. 511337

Fischer P B and Chou S Y 1993 J. Vac. Sci. Technol. B11 2524

Grossman H-J and Schubert E F 1993 Crit. Rev. Solid State Mater. Sci. 181

Hegde R I, Tobin P J, Reid K J, Maiti B and Ajuria S A 1995 Appl. Phys. Lett. 662882

Hibino H and Ogino T 1995 Appl. Phys. Lett. 67915

Ishizaka A and Shiraki Y 1986 J. Eletrochem. Soc. 133666

Iyer S S, Patton G L, Stork J M C, Meyerson B S and Harame D L 1989 IEEE Trans. Electron. Devices 362043

Kamath A, Kwong D L, Sun Y M, Blass P M, Whaley S and White J M 1997 Appl. Phys. Lett. 7063

LeGoues F K, Rosenberg R and Meyerson B S $1989 \mathrm{Appl}$. Phys. Lett. 54644
Liou H K, Mei P, Gennser U and Yang E S 1991 Appl. Phys. Lett. 591200

Lu H C, Gusev E P, Gustafsson T, Garfunkel E, Green M L, Brasen D and Feldman L C 1996 Appl. Phys. Lett. 692713

Nayak D, Kamjoo D, Woo J C S, Park J S and Wang K L 1990 Appl. Phys. Lett. 5666

Ogino T, Hibino H and Prabhakaran K 1996 J. Vac. Sci. Technol. B14 4234

Ogino T, Kobayashi Y, Prabhakaran K, Sumitomo K 1997 Mater. Res. Soc. Symp. Proc. (Pittsburgh: MRS) Vol. 438211

Park J S, Karunasiri R P G, Wang J L, Rhee S S and Chern C H 1989 Appl. Phys. Lett. 541564

Patton G L, lyer S S, Delage S L, Ganin E and Mcintosh R C 1988 Mater. Res. Soc. Symp. Proc. (Pittsburgh: MRS) Vol. 102295

People R 1986 IEEE J. Quantum Electron 221696

Prabhakaran K and Ogino T 1997 Surface Sci. 387 L1068

Prabhakaran K, Ogino T, Hull R C, Bean J C and Peticolas L C 1994a Surface Sci. 316 L1031

Prabhakaran K, Nishioka T, Kobayashi Y and Ogino T 1994b Appl. Surface Sci. 75341

Prabhakaran K, Nishioka T, Kobayashi Y and Ogino T 1993 Appl. Phys Lett 62864

Prabhakaran K, Ogino T, Scimeca T, Watanabe $Y$ and Oshima M 1994c Appl. Phys. Lett. 641839

Prabhakaran K, Sumitomo K and Ogino T 1996 Appl. Phys. Lett 681241

Prabhakaran K, Sumitomo K and Ogino T 1997a Appl. Phys. Lett. 70607

Prabhakaran K, Sumitomo K and Ogino T 1997b Appl. Surface Sci. 117/118 280

Prabhakaran K, Matsumoto T, Ogino T and Masumoto Y 1998 Appl. Phys. Lett. 723169

Shen T-C, Wang C, Abeln G C, Tucker J R, Lyding J W, Avouris Ph and Walkup R E 1995 Science 2681590

Skuja L 1994 J. Non-cryst. Solids 167229

Tabe M and Yamamoto T 1996 Appl. Phys. Lett. 692222

von Kanel H Materials Sci. Rep. 19928193

Yablonovitch E, Gmitter T J, Meade R D, Rappe A M, Brommer K D and Joannopoulos J D 1991 Phys. Rev. Lett. 673380 